\title{
DYNAMICS OF AN AUTONOMOUS SPACECRAFT CONTROL SYSTEM AT INITIAL TRANSITION TO A TRACKING MODE
}

\author{
Yevgeny Somov \\ Navigation, Guidance and Control \\ Samara State TechnicalUniversity \\ Russia \\ e_somov@mail.ru
}

\author{
Sergey Butyrin \\ Navigation, Guidance and Control \\ Samara State TechnicalUniversity \\ Russia \\ butyrinsa@mail.ru
}

\author{
Sergey Somov \\ Navigation, Guidance and Control \\ Samara State TechnicalUniversity \\ Russia \\ s_somov@mail.ru
}

Article history:

Received 21.10.2021, Accepted 25.11.2021

\begin{abstract}
The problems of autonomous digital control of the information satellites and space robots during their initial transition to a tracking mode, namely in the initial orientation modes, are considered. Autonomous angular guidance and modularly limited vector digital control using a vector of the modified Rodrigues parameters are applying to bring the spacecraft's orientation from completely arbitrary to the required one. The developed methods, algorithms and simulation results for a mini-satellite in a sun-synchronous orbit are presented.
\end{abstract}

\section{Key words}

Spacecraft, initial orientation modes, digital control.

\section{Introduction}

After separating any spacecraft (an information satellite [Testoyedov et al., 2017], a space robot [Somov et al., 2019] etc.) from a launch vehicle, the spacecraft (SC) begins to turn somersaults - to rotate with the angular velocity vector $\omega$ of arbitrary direction in its body reference frame (BRF) Oxyz. The first purpose of of the SC digital control is its initial transition to a given tracking mode. Here, the simplest task is the initial orientation modes (IOMs) of the SC attitude control system (ACS) when the $\mathrm{SC}$ orientation is bringed to a given position in the orbital reference frame (ORF) $\mathrm{O} x^{\mathrm{O}} y^{\mathrm{O}} z^{\mathrm{O}}$. If the SC is modeled as a rigid body, then indicated problems of its spatial angular motion are studied in theoretical mechanics [Aleksandrov and Tikhonov, 2018] and physics [Materassi and Morrison, 2018] as well as in mathematical control theory [Smirnov, 1981]. In this paper we consider the Earth-observing mini-satellite weighing of 250 $\mathrm{kg}$, which is separating from the launch vehicle in a sunsynchronous orbit with altitude $600 \mathrm{~km}$. We are assuming that considered miniature $\mathrm{SC}$ is equipped with the
ACS that contains a strapdown inertial navigation system (SINS) with correction by signals of GPS/GLONASS satellites and star trackers, cluster of the gyro angular rate sensors (ARSs), magnetometer (MM) and also the following drives: cluster of four flywheels - reaction wheels (RWs) under the scheme General Electric $(G E)$, see Fig. 1, and magnetic drive (MD).

We study the SC nonlinear control problems in the following IOMs: (i) the SC calm down in the inertial reference frame (IRF) at the ARS cluster's signals by the MD digital control while the angular velocity vector module $\omega \equiv|\boldsymbol{\omega}|>\omega_{1}^{*}$ at the specified value $\omega_{1}^{*}$; (ii) initialization of RW cluster, switching on it into the SC control loop and then the $\mathrm{SC}$ bringing to the required orientation in the ORF when the SINS signals are used; (iii) the SC angular stabilization in the ORF by autonomous digital control of the RW cluster with its unloading from the accumulated angular momentum (AM) using MD [Somov et al., 2018]. The problem of the SC autonomous angular guidance is solved while tracking the reference model for vector of the modified Rodrigues parameters (MRP) using a modularly limited digital control torque vector of the RW cluster at bringing the satellite orientation from arbitrary one to the required one in the ORF.

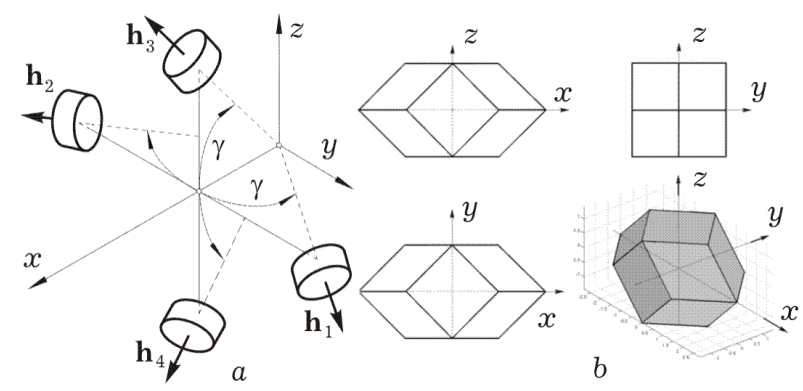

Figure 1. The $G E$ scheme ( $a$ ) and its AM variation domain $(b)$ 


\section{Mathematical Models and the Problem Statement}

In what follows, we use the notations $\{\cdot\} \equiv \operatorname{col}(\cdot)$, $[\cdot] \equiv \operatorname{line}(\cdot)$ for vectors with scalar $\langle\cdot, \cdot\rangle$, vector $(\{\cdot\} \times\{\cdot\})$ and dyad $[\{\cdot\} \cdot\{\cdot\}]$ products; for matrices $[\cdot \times],(\cdot)^{\mathrm{t}},\lceil\cdot\rfloor \equiv \operatorname{diag}(\cdot)$ and $\circ, \tilde{\sim}$ for quaternions $\boldsymbol{\Lambda}=$ $\left(\lambda_{0}, \boldsymbol{\lambda}\right), \boldsymbol{\lambda}=\left\{\lambda_{1}, \lambda_{2}, \lambda_{3}\right\}$, and also for the MRP vector $\boldsymbol{\sigma}=\mathbf{e} \operatorname{tg}(\Phi / 4)$ with Euler unit vector e and angle $\Phi$ of own rotation, $i=1,2 \ldots m \equiv 1 \div m$. The vector $\sigma$ is connected with quaternion $\boldsymbol{\Lambda}$ of the spacecraft BRF orientation in the IRF by relations $\boldsymbol{\sigma}=\boldsymbol{\lambda} /\left(1+\lambda_{0}\right)$ and $\lambda=2 \boldsymbol{\sigma} /\left(1+\sigma^{2}\right) ; \lambda_{0}=\left(1-\sigma^{2}\right) /\left(1+\sigma^{2}\right)$.

The model of the SC angular motion takes into account the flexibility of its structure and has the form

$$
\begin{gathered}
\dot{\mathbf{\Lambda}}=\mathbf{\Lambda} \circ \boldsymbol{\omega} / 2 ; \quad \mathbf{A}^{o}\{\dot{\boldsymbol{\omega}}, \ddot{\mathbf{q}}, \dot{\boldsymbol{\Omega}}\}=\left\{\mathbf{F}^{\omega}, \mathbf{F}^{q}, \mathbf{F}^{\mathrm{r}}\right\}, \\
\mathbf{F}^{\omega}=-\boldsymbol{\omega} \times \mathbf{G}+\mathbf{M}^{\mathrm{o}} ; \mathbf{F}^{q}=-\mathbf{A}^{q}\left(\mathbf{V}_{q} \dot{\mathbf{q}}+\mathbf{W}_{q} \mathbf{q}\right) ; \\
\mathbf{F}^{\mathrm{r}}=\mathbf{m}-\mathbf{m}^{\mathrm{f}} ; \boldsymbol{\omega}=\left\{\omega_{i}\right\} ; \mathbf{q}=\left\{q_{j}\right\} ; \boldsymbol{\Omega}=\left\{\Omega_{p}\right\} ; \\
i=1 \div 3 ; j=1 \div n^{q} ; p=1 \div 4 \\
\mathbf{A}^{o}=\left[\begin{array}{ccc}
\mathbf{J} & \mathbf{D}_{q} & \mathbf{D}_{r} \\
\mathbf{D}_{q}^{\mathrm{t}} & \mathbf{A}^{q} & \mathbf{0} \\
\mathbf{D}_{r}^{\mathrm{t}} & \mathbf{0} & \mathbf{A}^{r}
\end{array}\right] ; \mathbf{A}_{\gamma}=\left[\begin{array}{cccc}
C_{\gamma} & C_{\gamma} & C_{\gamma} & C_{\gamma} \\
S_{\gamma} & -S_{\gamma} & 0 & 0 \\
0 & 0 & S_{\gamma}-S_{\gamma}
\end{array}\right] ; \\
\mathbf{D}_{r}=J_{r} \mathbf{A}_{\gamma}, \mathbf{A}^{r}=J_{r} \mathbf{I}_{4} ; \quad C_{\gamma} \equiv \cos \gamma, S_{\gamma} \equiv \sin \gamma ;
\end{gathered}
$$

matrix $\mathbf{A}_{\gamma}$ composed of unit vectors of the RW's axes; $\mathbf{M}^{\mathrm{o}} \equiv \mathbf{M}^{\mathrm{m}}+\mathbf{M}^{\mathrm{d}} ; \mathbf{G}=\mathbf{G}^{\mathrm{o}}+\mathbf{D}_{q} \dot{\mathbf{q}}$ is the AM vector of whole mechanical system, $\mathbf{G}^{\circ}=\mathbf{K}+\mathcal{H}, \mathbf{K}=\mathbf{J} \boldsymbol{\omega}$; the columns $\mathcal{H}=\left\{\mathrm{H}_{i}\right\}$ and $\mathbf{h} \equiv\left\{\mathrm{h}_{p}\right\}, \mathrm{h}_{p} \equiv J_{r} \Omega_{p}$ represent AMs of the RW cluster and individual RWs related by ratio $\left.\mathcal{H}=\mathbf{A}_{\gamma} \mathbf{h} ; \mathbf{A}^{q}=\left\lceil\mu_{j}\right\rfloor ; \mathbf{V}_{q}=\left\lceil\frac{\delta^{q}}{\pi} \Omega_{j}^{q}\right\rfloor ; \mathbf{W}_{q}=\left\lceil\Omega_{j}^{q}\right)^{2}\right\rfloor ;$ a vector of the MD torque is $\mathbf{M}^{\mathrm{m}}=\left\{m_{i}^{\mathrm{m}}\right\}=-\mathbf{L} \times \mathbf{B}$, where vector of electromagnetic moment (EMM) $\mathbf{L}=$ $\left\{l_{i}\right\}$ with components $\left|l_{i}\right| \leq 1^{\mathrm{m}}$ and the induction vector of the Earth magnetic field (EMF) $\mathbf{B}=\mathrm{Bb}$ with unit vector $\mathbf{b}$ are defined in BRF; the columns $\mathbf{m}=\left\{m_{p}\right\}$ and $\mathbf{m}^{\mathrm{f}}=\left\{m_{p}^{\mathrm{f}}\right\}$ represent the control torques and torques of dry friction forces along the axes of the RW rotation, and vector $\mathbf{M}^{\mathrm{d}}$ - external disturbing torques.

The values of control torque and own AM for each RW are limited, $\left|m_{p}(t)\right| \leq \mathrm{m}^{\mathrm{m}} ;\left|\mathrm{h}_{p}(t)\right| \leq \mathrm{h}^{\mathrm{m}}$. Further on, for the RW cluster we use the torque vector $\mathbf{M}^{\mathrm{r}} \equiv\left\{\mathrm{M}_{i}^{\mathrm{r}}\right\}=-\mathcal{H}^{*}=-\mathbf{A}_{\gamma} \dot{\mathbf{h}}$ with the symbol $(\cdot)^{*}$ of a local time derivative.

When the SC with RW cluster is considered as a free solid $\left(\mathbf{M}^{\circ}=\mathbf{0}\right)$ and the ACS has a balance by its total $\mathrm{AM}$ vector $(\mathbf{G} \equiv \mathbf{0})$, the $\mathrm{SC}$ angular motion in inertial reference frame (IRF) is described by the model

$$
\dot{\Lambda}=\boldsymbol{\Lambda} \circ \boldsymbol{\omega} / 2 ; \dot{\boldsymbol{\omega}}=\mathbf{J}^{-1} \mathbf{M}^{\mathrm{r}}=\boldsymbol{\varepsilon} \equiv \mathbf{u} .
$$

At applying the MPR vector $\boldsymbol{\sigma}$ this kinematic model of the SC angular motion takes the form

$$
\dot{\boldsymbol{\sigma}}=\left(1-\sigma^{2}\right) \boldsymbol{\omega} / 4+(\boldsymbol{\sigma} \times \boldsymbol{\omega}+\boldsymbol{\sigma}\langle\boldsymbol{\sigma}, \boldsymbol{\omega}\rangle) / 2 ; \dot{\boldsymbol{\omega}}=\mathbf{u}
$$

with initial conditions $\boldsymbol{\sigma}\left(t_{\mathrm{o}}\right)=\boldsymbol{\sigma}_{\mathrm{o}}, \boldsymbol{\omega}\left(t_{\mathrm{o}}\right)=\boldsymbol{\omega}_{\mathrm{o}}$ and the vector $\sigma_{\mathrm{o}} \equiv \mathbf{e}_{\mathrm{o}} \operatorname{tg}\left(\Phi_{\mathrm{o}} / 4\right)$ is arbitrary when $\left|\Phi_{\mathrm{o}}\right|<2 \pi$.

For the SC guidance law $\Lambda^{p}, \boldsymbol{\omega}^{p}, \varepsilon^{p}=\dot{\boldsymbol{\omega}}^{p}$ in the IRF the error quaternion $\mathbf{E}=\left(e_{0}, \mathbf{e}\right)=\tilde{\mathbf{\Lambda}}^{p} \circ \boldsymbol{\Lambda}$ corresponds to Euler parameters' vector $\mathcal{E}=\left\{e_{0}, \mathbf{e}\right\}$ with $\mathbf{e}=\left\{e_{i}\right\}$, the angular error matrix $\mathbf{C}^{\mathrm{e}} \equiv \mathbf{C}(\mathcal{E})=\mathbf{I}_{3}-2[\mathbf{e} \times] \mathbf{Q}_{\mathrm{e}}^{\mathrm{t}}$ with the matrix $\mathbf{Q}_{\mathrm{e}} \equiv \mathbf{Q}(\mathcal{E})=\mathbf{I}_{3} e_{0}+[\mathbf{e} \times]$, the MRP vector $\sigma^{\mathrm{e}}=\mathbf{e}^{\mathrm{e}} \operatorname{tg}\left(\Phi^{\mathrm{e}} / 4\right)$ and the angular error vector $\delta \boldsymbol{\phi}=\left\{\delta \phi_{i}\right\}=2\left\{e_{o} e_{i}\right\}$. The error vector $\delta \boldsymbol{\omega} \equiv \boldsymbol{\omega}^{\mathrm{e}}$ in angular rate is calculated by the ratio $\boldsymbol{\omega}^{\mathrm{e}}=\boldsymbol{\omega}-\mathbf{C}^{\mathrm{e}} \boldsymbol{\omega}^{p}$.

Assume that a discrete measuring of the SC orientation $\boldsymbol{\Lambda}_{l} \equiv \boldsymbol{\Lambda}\left(t_{l}\right)$ is performed by the SINS with period $T_{p}$, $l \in \mathbb{N}_{0} \equiv[0,1,2, \ldots)$. Let's also assume that at the time moments $t_{k}, k \in \mathbb{N}_{0}$ with the period $T_{u}$, the RW digital control is formed, and the MD digital control acts when $t \in\left[t_{r}, t_{r+1}\right), r \in \mathbb{N}_{0}$ with a period $T_{u}^{\mathrm{m}} \gg T_{u}$.

In this paper we research the following problems:

(i) development of discrete algorithms for digital control of both the MD and the RW cluster;

(ii) synthesis of a nonlinear digital control law $\mathbf{u}_{k} \equiv$ $\mathbf{u}\left(\boldsymbol{\sigma}_{k}, \boldsymbol{\omega}_{k}\right)$ for the reference model (2) \& (3) with the bounded modules of both control and angular velocity vectors, which provides asymptotic stability of the closed nonlinear continuous-discrete reference model; (iii) synthesis of nonlinear digital control law for the RW cluster, which after completing the satellite's calming down mode provides the SC transition to its required angular position in the ORF;

(iv) computer simulation of the ACS performance in the IOMs for mini-satellite in a sun-synchronous orbit when using the $\mathrm{SC}$ autonomous angular guidance.

\section{Digital Control of Magnetic Drive}

When the $\mathrm{SC}$ is modelled as a solid $\left(\mathbf{M}^{\mathrm{d}}=\mathbf{0} ; \mathbf{M}^{r}=\mathbf{0}\right.$ and $\mathbf{G}=\mathbf{K}$ ) the model of the SC rotation dynamics in (1) is presented in the form $\dot{\mathbf{K}}+\boldsymbol{\omega} \times \mathbf{K}=\mathbf{M}$ where $\dot{\mathbf{K}} \equiv$ $\mathbf{K}^{*}=\mathbf{J} \dot{\omega}$ and external control torque $\mathbf{M}=\mathbf{M}^{\mathrm{m}}$. For synthesis of the locally optimal continuous control laws $\mathbf{M}=\mathbf{M}(\boldsymbol{\omega})$ we used Lyapunov function $\mathrm{v}=\langle\mathbf{K}, \mathbf{K}\rangle$. As a result, we have established that during the $\mathrm{SC}$ calm down with minimum coercion $\mathrm{M}^{2}=\|\mathbf{M}\|^{2}$ a control law has the form $\mathbf{M}=-a \mathrm{Kk}$ with the AM unit vector $\mathbf{k}=$ $\mathbf{K} / \mathrm{K}$ and parameter $a>0$, and control law $\mathbf{M}=-m \mathbf{k}$, with a module bounded by a parameter $m>0$, represents the time-optimal control torque.

Assume that in the time moments $t_{r}=r T_{r}^{\mathrm{m}}, r \in$ $\mathbb{N}_{0}$ the EMF induction vector $\mathbf{B}_{r}=\mathrm{B}_{r} \mathbf{b}_{r}$ and vector $\boldsymbol{\omega}_{r}$ are measured by the MM and the ARS cluster. At formation of the command $\mathbf{M}_{r}=-a \mathbf{K}_{r}$ for the MD torque vector on each time semi-interval $t \in\left[t_{r}, t_{r+1}\right)$ with period $T_{r}^{\mathrm{m}}$, first, we define vector $\mathbf{M}_{r}^{p}$ of required variation for a pulse of control torque

$$
\mathbf{M}_{r}^{p} \equiv \int_{t_{r}}^{t_{r+1}} \mathbf{K}(\tau) d \tau=-\mathbf{K}_{r}\left(1-\exp \left(-a T_{r}^{\mathrm{m}}\right)\right) \mathbf{k}_{r}
$$

with its presentation $\mathbf{M}_{r}^{p}=\mathbf{b}_{r} \times\left(\mathbf{M}_{r}^{p} \times \mathbf{b}_{r}\right)+\mathbf{b}_{r}\left\langle\mathbf{M}_{r}^{p}, \mathbf{b}_{r}\right\rangle$. Then we assign value $\mathbf{M}_{r}^{p \mathrm{~m}}=\mathbf{b}_{r} \times\left(\mathbf{M}_{r}^{p} \times \mathbf{b}_{r}\right)$ with condition $\mathbf{b}_{r}\left\langle\mathbf{M}_{r}^{p}, \mathbf{b}_{r}\right\rangle=\mathbf{0}$, that ensures the MD energy saving. Next, vector of required variation for a pulse of control torque $\mathbf{M}_{r}^{p \mathrm{~m}} \equiv-\Delta \mathrm{I}_{r}^{\mathrm{m}} \mathbf{k}_{r}$ with module $\Delta \mathrm{I}_{r}^{\mathrm{m}}=\mathrm{K}_{r}\left(1-\exp \left(-a T_{r}^{\mathrm{m}}\right)\right)$ and unit vector $\mathbf{k}_{r}$ is applied to form the EMM digital control vector $\mathbf{L}_{r}=\left\{l_{i r}\right\}$ 


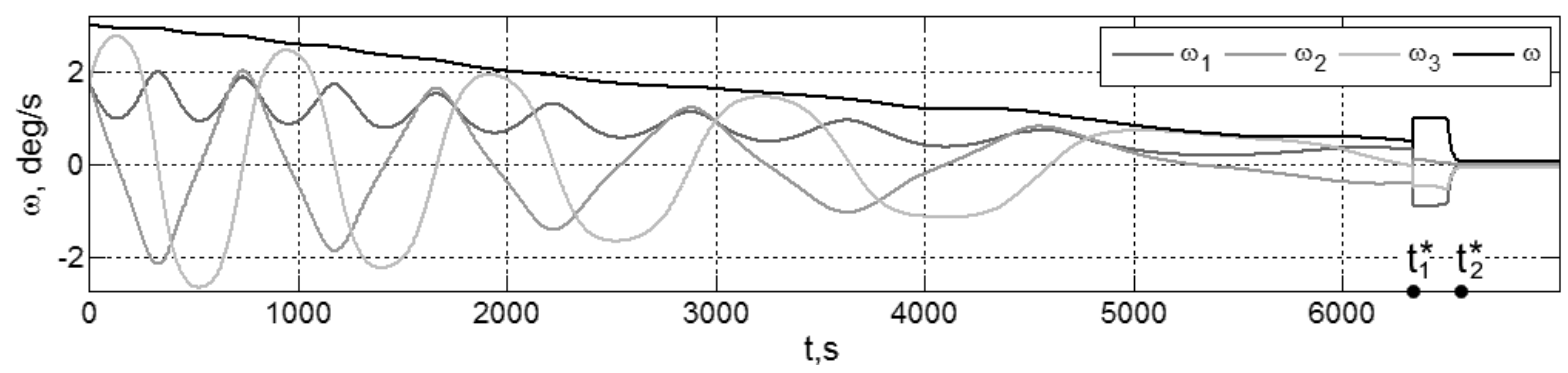

Figure 2. Changing the SC angular velocity vector at digital control by the MD and RW cluster in the IOMs

for magnetic drive. Moreover, a mutual orientation is defined for the unit vectors $\mathbf{b}_{r}$ and $\mathbf{k}_{r}$ in the BRF. If $\left|\left\langle\mathbf{b}_{r}, \mathbf{k}_{r}\right\rangle\right|>\cos (\pi / 3)$ than the MD is not switched on at the current control period, otherwise vector $\mathbf{L}_{r}=\left\{l_{i r}\right\}$ with restricted components $\left|l_{i r}\right| \leq 1^{\mathrm{m}}$ is formed by explicit relation $\mathbf{L}_{r}=\left(\Delta \mathrm{I}_{r}^{\mathrm{m}} / T_{r}^{\mathrm{m}}\right)\left(\mathbf{b}_{r} \times \mathbf{k}_{r}\right) / \mathrm{B}_{r}$. That algorithm is applied also for the RW cluster unloading from accumulated angular momentum.

\section{Digital Control of the RW Cluster}

Normalized to $\mathrm{h}^{\mathrm{m}}$ form the RW's AM vector $\mathbf{h}=$ $\left\{h_{p}\right\}$ with $h_{p}=\mathrm{h}_{p} / \mathrm{h}^{\mathrm{m}},\left|h_{p}\right| \leq 1$ is applied for computing the RW cluster normed AM as follows

$$
\begin{gathered}
\boldsymbol{h} \equiv \boldsymbol{H} / \mathrm{h}^{\mathrm{m}}=\{x, y, z\}=\left\{x_{1}+x_{2}, S_{\gamma} h_{12}^{-}, S_{\gamma} h_{34}^{-}\right\}, \\
x_{1}=C_{\gamma} h_{12}^{+}, x_{2}=C_{\gamma} h_{34}^{+} ; h_{12}^{ \pm}=h_{1} \pm h_{2}, h_{34}^{ \pm}=h_{3} \pm h_{4} .
\end{gathered}
$$

For the RW cluster, the fundamental problem is simultaneous distribution of its AM $\mathcal{H}$ and control torque $\mathbf{M}^{r}=-\mathcal{H}^{*}$ vectors between four RWs. Main idea of the employed explicit distribution law consists in achieving the strict uniformity in terms of the saturation resources for the first and second RW pairs. In the normalized form, the law is described by the relations

$$
\begin{gathered}
f_{\rho}(\mathbf{h})=\tilde{x}_{1}-\tilde{x}_{2}+\rho\left(\tilde{x}_{1} \tilde{x}_{2}-1\right)=0 ; \\
\dot{f}_{\rho}(\mathbf{h})=\left\langle\mathbf{a}^{\mathrm{f}}(\mathbf{h}), \mathbf{h}\right\rangle=\Phi_{\rho}\left(f_{\rho}(\mathbf{h})\right) \equiv-\operatorname{sat}\left(\mu_{\rho} f_{\rho}(\mathbf{h}), \phi_{\rho}\right), \\
\text { where } \tilde{x}_{1} \equiv x_{1} / q_{y} ; \tilde{x}_{2} \equiv x_{2} / q_{z} ; q_{s}=\left(4 C_{\gamma}^{2}-s^{2}\right)^{1 / 2}, \\
s=y, z ; 0<\rho<1 \text { and } \phi_{\rho}, \mu_{\rho}, \rho \text { are positive para- } \\
\text { meters, and column } \mathbf{a}^{\mathrm{f}}(\mathbf{h})=\left\{a_{p}^{\mathrm{f}}\right\}=\partial f_{\rho}(\mathbf{h}) / \partial \mathbf{h} \text { has } \\
\text { the components } \\
a_{1,2}^{\mathrm{f}}=2 C_{\gamma}\left(2 C_{\gamma}^{2} \pm S_{\gamma}^{2} h_{2} h_{12}^{-}\right)\left(1+\rho C_{\gamma} h_{34}^{+} / q_{z}\right) / q_{y}^{3} ; \\
a_{3,4}^{\mathrm{f}}=2 C_{\gamma}\left(2 C_{\gamma}^{2} \mp S_{\gamma}^{2} h_{4} h_{34}^{-}\right)\left(1+\rho C_{\gamma} h_{12}^{+} / q_{y}\right) / q_{z}^{3} .
\end{gathered}
$$

With notation $q_{y z}^{ \pm} \equiv q_{y} \pm q_{z}, b \equiv x / 2, c \equiv q_{y} q_{z}-b^{2}$ the normed AM vector $\boldsymbol{h}=\{x, y, z\}$ is distributed as per condition $f_{\rho}(\mathbf{h})=0$ firstly among RW pairs by the relation $x_{1}=(x+\Delta) / 2, x_{2}=(x-\Delta) / 2$, where

$$
\Delta \equiv\left(q_{y z}^{+} / \rho\right)\left(1-\left(1-4 \rho\left[q_{y z}^{-} b+\rho c\right] /\left(q_{y z}^{+}\right)^{2}\right)^{1 / 2}\right),
$$

and next among two RWs in each pair by evident relations. To define column $\mathbf{m}$, the relation $\mathbf{A}_{\gamma} \dot{\mathbf{h}}=\dot{\boldsymbol{h}}$ is supplemented with equation $\left\langle\mathbf{a}^{\mathrm{f}}(\mathbf{h}), \dot{\mathbf{h}}\right\rangle=\Phi_{\rho}\left(f_{\rho}(\mathbf{h})\right)$. As a result, we obtain four linear equations and the column $\mathbf{m}$ of the RW cluster control torques is calculated as $\mathbf{m} \equiv\left\{m_{p}\right\}=\left\{\mathbf{A}_{\gamma},\left[\mathbf{a}_{p}^{\mathrm{f}}\right]\right\}^{-1}\left\{-\mathbf{M}^{\mathrm{r}}, \mathrm{h}^{\mathrm{m}} \Phi_{\rho}\left(f_{\rho}(\mathbf{h})\right)\right\}$.

The RW cluster is unloading from the accumulated
AM using a direct compensating scheme: simultaneous formed digital command vectors $\mathbf{L}_{r}$ to magnetic drive and $\mathbf{M}_{k}^{c}$ to the RW cluster are equivalent in the torque pulse and have opposite signs, taking into account the multiplicity of periods $T_{u}^{\mathrm{m}}$ and $T_{u}$. Therefore, the preliminary digital control vector $\tilde{\mathbf{m}}_{k}$ of the RW cluster is calculated in the form

$$
\tilde{\mathbf{m}}_{k}=\left\{\mathbf{A}_{\gamma},\left[\mathbf{a}_{k}^{\mathrm{f}}\right]\right\}^{-1}\left\{-\left(\mathbf{M}_{k}^{\mathrm{r}}+\mathbf{M}_{k}^{\mathrm{c}}\right), \mathrm{h}^{\mathrm{m}} \Phi_{\rho}\left(f_{\rho}\left(\mathbf{h}_{k}\right)\right)\right\} .
$$

Each RW has a built-in micro-processor for identification of the RW dry friction torque, here we consider only one RW rotation axis, without index $p$.

The simplest approximate model of the RW rotation is presented in form $\dot{\Omega}(t)=a(t)-a^{\mathrm{f}}(t)$, where the control acceleration $a(t)=m(t) / J_{r}$ and acceleration $a^{\mathrm{f}}(t)=a_{\mathrm{o}}^{\mathrm{f}} \operatorname{sign}(\Omega(t)) \in\left[-a_{\mathrm{o}}^{\mathrm{f}}, a_{\mathrm{o}}^{\mathrm{f}}\right]$ describes the dry friction torque, moreover for known RW moment of inertia $J_{r}$ the parameter $a_{\mathrm{o}}^{\mathrm{f}}=m_{\mathrm{o}}^{\mathrm{f}} / J_{r}=$ const is unknown.

Assuming that $a^{\mathrm{f}}(t)=a_{s}^{\mathrm{f}}=$ const $\forall t \in\left[t_{s}, t_{s+1}\right), s \in$ $\mathbb{N}_{0}$, the discrete Luenberger observer

$$
\begin{gathered}
\hat{\Omega}_{s+1}=\hat{\Omega}_{s}+\left(a_{s}-\hat{a}_{s}^{\mathrm{f}}\right) T_{q}+g_{1}^{\mathrm{f}} \delta \Omega_{s} ; \\
\hat{a}_{s+1}^{\mathrm{f}}=\hat{a}_{s}^{\mathrm{f}}+g_{2}^{\mathrm{f}} \delta \Omega_{s} ; \delta \Omega_{s+1}=\Omega_{s+1}-\hat{\Omega}_{s+1}
\end{gathered}
$$

with period $T_{q} \ll T_{u}$ is applied when the RW rotation velocity $\Omega(t)$ changes only in a small neighborhood of its value $\Omega=0$ for obtaining an estimate $\hat{a}_{s}^{\mathrm{f}}$, where parameters $g_{1}^{\mathrm{f}}$ and $g_{2}^{\mathrm{f}}$ are defined by explicit relations.

In this case, a discrete estimation of the RW dry friction torque has the form $\hat{m}_{s}^{\mathrm{f}}=J_{r} \hat{a}_{s}^{\mathrm{f}}$. This allows to obtain the estimations $\hat{m}_{k}^{\mathrm{f}}(t) \forall t \in\left[t_{k}, t_{k+1}\right), k \in \mathbb{N}_{0}$ taking into account the multiplicity of periods $T_{u}$ and $T_{q}$. Approximate compensation of the RW's dry friction torques is implemented by using the vectors $\tilde{\mathbf{m}}_{k}=$ $\left\{\tilde{m}_{p k}\right\}$ and $\hat{\mathbf{m}}_{k}^{\mathrm{f}}=\left\{\hat{m}_{p k}^{\mathrm{f}}\right\}$. As a result, the final vector $\mathbf{m}_{k}$ of the RW cluster digital control is formed by relation $\mathbf{m}_{k}=\tilde{\mathbf{m}}_{k}+\hat{\mathbf{m}}_{k}^{\mathrm{f}}$.

\section{The Reference Guidance Model}

Direct and inverse kinematic equations for the MRP vector $\sigma$ are represented in the form $\dot{\sigma}=\mathbf{B}(\sigma) \omega$ and $\boldsymbol{\omega}=\mathbf{D}(\boldsymbol{\sigma}) \dot{\boldsymbol{\sigma}}$ with the matrices

$$
\begin{aligned}
& \mathbf{B}(\boldsymbol{\sigma}) \equiv \frac{1}{4}\left(1-\sigma^{2}\right) \mathbf{I}_{3}+\frac{1}{2}([\boldsymbol{\sigma} \times]+[\boldsymbol{\sigma} \cdot \boldsymbol{\sigma}]) ; \\
& \mathbf{D}(\boldsymbol{\sigma}) \equiv \mathbf{B}^{-1}(\boldsymbol{\sigma})=\left(8 /\left(1+\sigma^{2}\right)^{2}\right) \mathbf{B}^{\mathrm{t}}(\boldsymbol{\sigma}) .
\end{aligned}
$$

Compact notation of second derivative of function $\sigma$

$$
\begin{aligned}
\ddot{\boldsymbol{\sigma}}= & (1 / 2)\left[-\langle\boldsymbol{\sigma}, \dot{\boldsymbol{\sigma}}\rangle \boldsymbol{\omega}+(1 / 2)\left(1-\sigma^{2}\right) \boldsymbol{\varepsilon}+\dot{\boldsymbol{\sigma}} \times \boldsymbol{\omega}\right. \\
& +\boldsymbol{\sigma} \times \boldsymbol{\varepsilon}+\dot{\boldsymbol{\sigma}}\langle\boldsymbol{\sigma}, \boldsymbol{\omega}\rangle+\boldsymbol{\sigma}\langle\dot{\boldsymbol{\sigma}}, \boldsymbol{\omega}\rangle+\boldsymbol{\sigma}\langle\boldsymbol{\sigma}, \boldsymbol{\varepsilon}\rangle]
\end{aligned}
$$




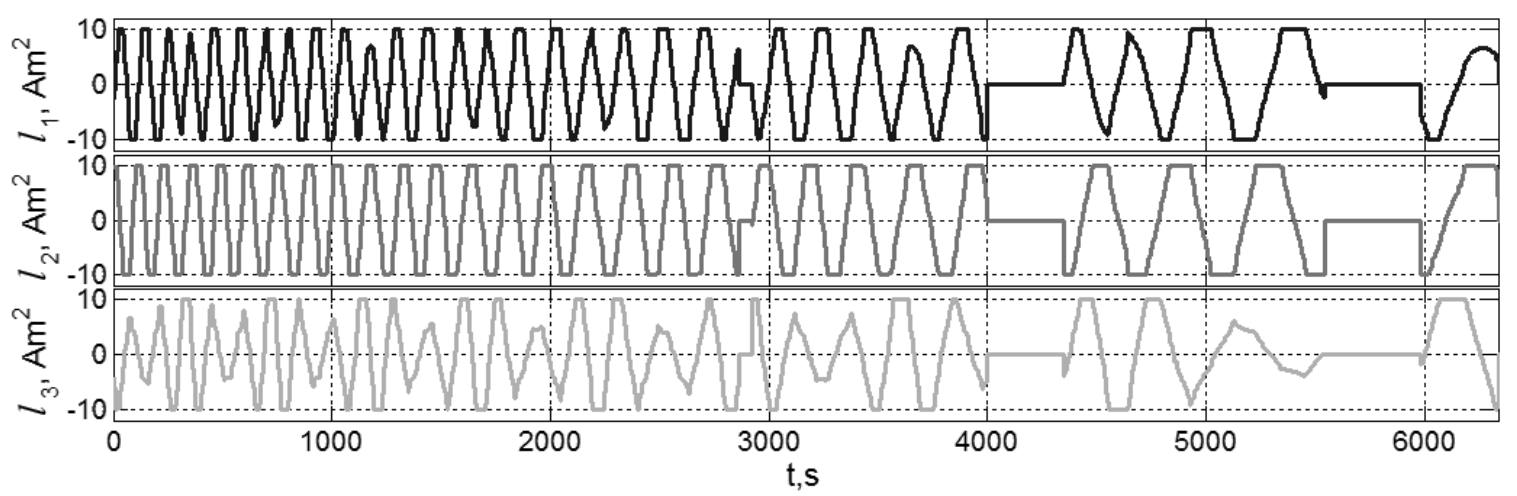

Figure 3. Changing the EMM vector at the MD digital control in the mini-satellite's calm down mode

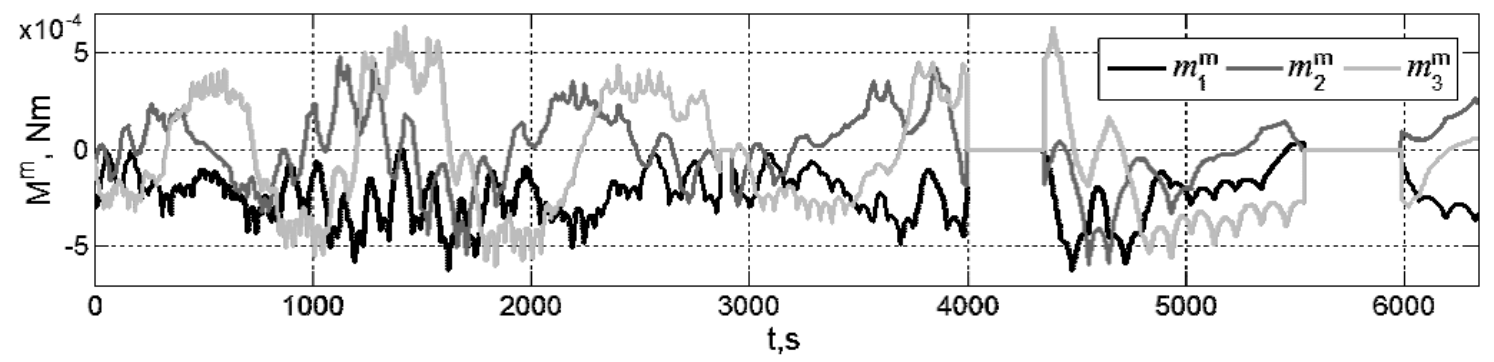

Figure 4. Changing the MD torque vector at its digital control in the mini-satellite's calm down mode

brings the controlled part of the reference guidance model to the form $\ddot{\sigma}=\mathbf{v} \equiv \mathbf{b}(\sigma, \omega)+\mathbf{B}(\boldsymbol{\sigma}) \mathbf{u}$ with function $\mathbf{b}(\boldsymbol{\sigma}, \boldsymbol{\omega})=([(\mathbf{B}(\boldsymbol{\sigma}) \boldsymbol{\omega}) \times]+[\boldsymbol{\sigma} \cdot \mathbf{B}(\boldsymbol{\sigma}) \boldsymbol{\omega}]) \boldsymbol{\omega} / 2$.

Using the methods of feedback linearization, modal synthesis and vector Lyapunov functions [Somov, 1996] for model $\ddot{\sigma}=\mathbf{v}$ on a single desired spectrum $S_{*}=$ $-\alpha \pm j \beta$ with $j=\sqrt{-1}$, we have obtained the control law $\mathbf{v}(\boldsymbol{\sigma}, \dot{\boldsymbol{\sigma}})=-\left(k_{\sigma} \boldsymbol{\sigma}+k_{\omega} \mathbf{B}(\boldsymbol{\sigma}) \boldsymbol{\omega}\right)$. In discrete form with period $T_{u}$ this control law is represented as $\mathbf{v}_{k}=-\left(k_{\sigma}^{d} \boldsymbol{\sigma}_{k}+k_{\omega}^{d} \mathbf{B}\left(\boldsymbol{\sigma}_{k}\right) \boldsymbol{\omega}_{k}\right)$. Here, for a given normalized regulation time $T_{r}$, the coefficients $k_{\sigma}^{d}$ and $k_{\omega}^{d}$ are calculated using explicit relations

$\omega_{*}=3 /\left(\xi T_{r}\right) ; \alpha=\xi \omega_{*}, \beta=\omega_{*}\left(1-\xi^{2}\right)^{1 / 2} ;$

$a_{1}=-2 \exp \left(-\alpha T_{u}\right) \cos \left(\beta T_{u}\right), a_{2}=\exp \left(-2 \alpha T_{u}\right)$;

$k_{\sigma}^{d}=\left(1+a_{1}+a_{2}\right) / T_{u}^{2}, k_{\omega}^{d}=\left(3+a_{1}-a_{2}\right) /\left(2 T_{u}\right)$,

which are fair $\forall \xi>0$. Preliminary control law is defined as $\tilde{\mathbf{u}}(\boldsymbol{\sigma}, \boldsymbol{\omega}) \equiv\left\{\tilde{u}_{i k}\right\}=\mathbf{D}(\boldsymbol{\sigma})(\mathbf{v}-\mathbf{b}(\boldsymbol{\sigma}, \boldsymbol{\omega}))$, that provides uniform asymptotic stability of trivial solution for model (3), and its discrete form is represented as

$$
\tilde{\mathbf{u}}_{k}=-\left[\mathbf{D}\left(\boldsymbol{\sigma}_{k}\right)\left(k_{\sigma}^{d} \boldsymbol{\sigma}_{k}+\mathbf{b}\left(\boldsymbol{\sigma}_{k}, \boldsymbol{\omega}_{k}\right)\right)+k_{\omega}^{d} \boldsymbol{\omega}_{k}\right] .
$$

When digital control is finally formed at the time $t_{k}$, the restrictions on control vector module $\left(|\mathbf{u}| \equiv u \leq \mathrm{u}^{\mathrm{m}}\right)$ and angular velocity vector module $\left(|\boldsymbol{\omega}| \equiv \omega \leq \omega^{\mathrm{m}}\right)$ are taken into account in (4) according to the following simple algorithm A:

1) by the value of digital control $\tilde{\mathbf{u}}_{k}(4)$ at the time $t_{k}$, there is calculated predictive value of angular velocity vector $\boldsymbol{\omega}_{k}^{q}=\boldsymbol{\omega}_{k}+\tilde{\mathbf{u}}_{k} T_{u}$ achieved at the end of the time interval duration $T_{u}$, and if $\left|\boldsymbol{\omega}_{k}^{q}\right|>\omega^{\mathrm{m}}$ then control vector $\tilde{\mathbf{u}}_{k}$ is redefined as $\tilde{\mathbf{u}}_{k}=\left(\omega^{\mathrm{m}}\left(\boldsymbol{\omega}_{k}^{q} / \omega_{k}^{q}\right)-\boldsymbol{\omega}_{k}\right) / T_{u}$;

2) next, if $\left|\tilde{\mathbf{u}}_{k}\right| \equiv \tilde{u}_{k}>\mathrm{u}^{\mathrm{m}}$ then vector $\mathbf{u}_{k}=\mathrm{u}^{\mathrm{m}} \tilde{\mathbf{u}}_{k} / \tilde{u}_{k}$ $\forall t \in\left[t_{k}, t_{k+1}\right)$ is formed, otherwise vector $\mathbf{u}_{k}=\tilde{\mathbf{u}}_{k}$.
Checking the operability of this digital control law was presented in our article [Somov et al., 2021].

\section{Autonomous Digital Control}

The autonomous guidance and digital attitude control of the mini-satellite is based on analytical relations linking the required coordinates of the SC state with the measured coordinates of its angular movement.

Considered problem consists in the synthesis of the SC autonomous control laws in the initial orientation modes, including the SC bringing from an arbitrary orientation in the IRF to the specified orientation in the ORF, for simplicity coinciding with this reference frame. In this case, at quaternion $\Lambda^{\circ}$ of the BRF orientation in the IRF we obtain the SC guidance law $\Lambda^{p}=\Lambda^{\circ}, \boldsymbol{\omega}^{p}=\boldsymbol{\omega}^{\circ}$ and $\varepsilon^{p}=\varepsilon^{\circ}$. In the ORF $\mathrm{O} x^{\circ} y^{\circ} z^{\mathrm{o}}$ the $\mathrm{SC}$ orientation is defined also by the Euler-Krylov angles $\phi_{1}$ (roll), $\phi_{2}$ (yaw) and $\phi_{3}$ (pitch) in the sequence 312 of elementary turns, that make up the column $\phi \equiv\left\{\phi_{i}\right\}$ and the matrix $\mathrm{C}^{\mathrm{O}}(\phi)=\mathrm{C}^{\mathrm{e}} \equiv \mathbf{C}(\mathcal{E})$.

All kinematic parameters $\left(\Lambda^{\circ}, \omega^{\circ}\right.$ and $\left.\varepsilon^{\circ}\right)$ of the ORF angular motion in the IRF are formed directly onboard

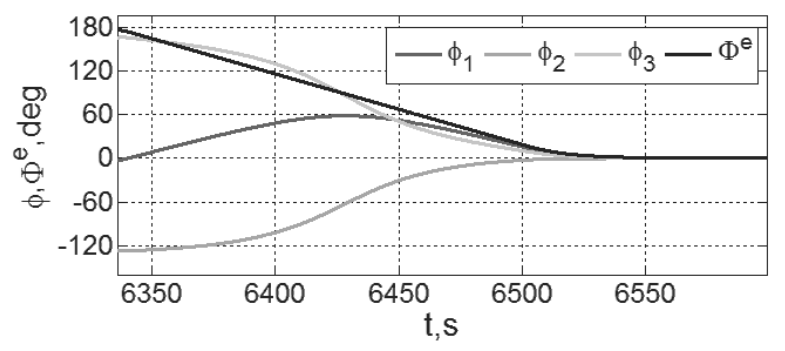

Figure 5. The angles $\phi_{i}$ and $\Phi^{\mathrm{e}}$ of the SC rotation in ORF 


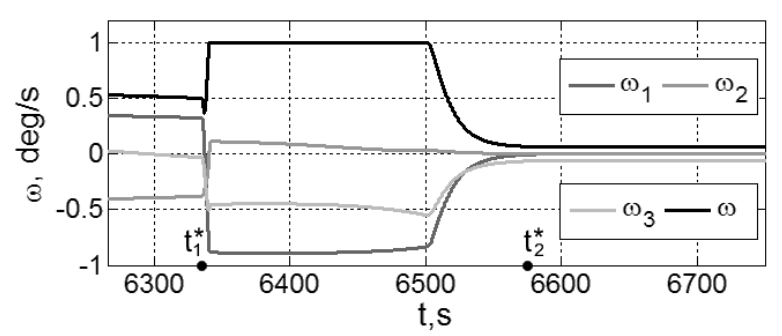

Figure 6. Changing the vector $\boldsymbol{\omega}$ at bringing the SC to ORF

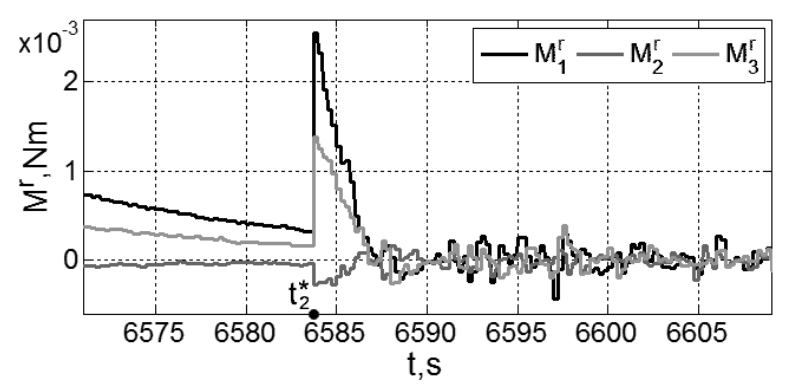

Figure 7. The RW cluster control torques at the ACS switching

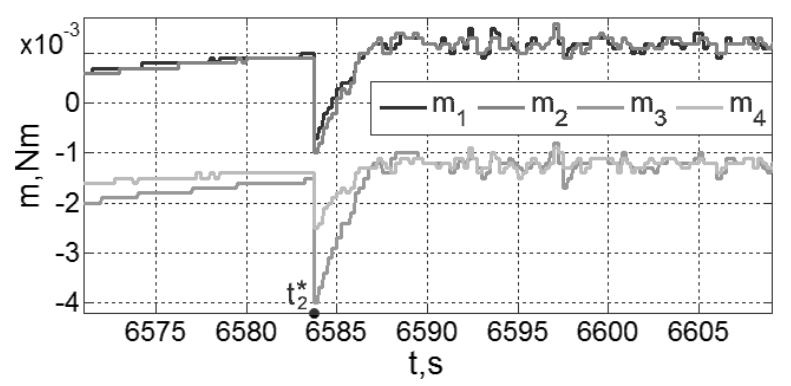

Figure 8. The RW's control torques at the ACS switching

mini-satellite, first with period $T_{u}^{\mathrm{m}}$ during the SC calm down in the IRF and then with period $T_{u}$ based on the methods of a filtering, approximation and interpolation [Testoyedov et al., 2017].

On the other hand, quaternion $\mathbf{\Lambda}$ of the SC actual orientation in the IRF and vector $\boldsymbol{\omega}$ of its angular velocity are measured by the SINS and ARS cluster, so we have an opportunity for the SC autonomous control in the IOMs.

Assume that SC separates from a booster at the time $t_{0}=0$ and the SC angular rate vector $\boldsymbol{\omega}(t)$ takes the value $\boldsymbol{\omega}_{0}=\boldsymbol{\omega}\left(t_{0}\right)$ with an arbitrary quaternion $\boldsymbol{\Lambda}_{0}=\boldsymbol{\Lambda}\left(t_{0}\right)$ of its orientation in the IRF. Then the vector of digital EMM $\mathbf{L}_{r}=\left\{l_{i r}\right\}, l_{i r}=$ const $\forall t \in\left[t_{r}, t_{r}+T_{u}^{\mathrm{m}}\right)$ with $\left|l_{i r}\right| \leq \mathrm{l}^{\mathrm{m}}$ begins to form automatically using measurements only of the magnetometer and the ARS cluster. As a result, the vector $\mathbf{M}_{r}^{\mathrm{m}}(t)=\left\{m_{i r}^{\mathrm{m}}(t)\right\} \forall t \in\left[t_{r}, t_{r}+T_{u}^{\mathrm{m}}\right), r \in$ $\mathbb{N}_{0}$ is generated to slow downing the $\mathrm{SC}$ rotation in the IRF and the SC's angular calming mode ends when the condition $|\boldsymbol{\omega}(t)| \leq \omega_{1}^{*}$ is met for a given value $\omega_{1}^{*}=$ $\left|\boldsymbol{\omega}\left(t_{1}^{*}\right)\right|$ at any time moment $t=t_{1}^{*}$.

At the same time moment the values $\boldsymbol{\Lambda}_{* 1}=\boldsymbol{\Lambda}\left(t_{1}^{*}\right)$ and $\boldsymbol{\omega}_{1}^{*}=\boldsymbol{\omega}\left(t_{1}^{*}\right)$ are measured by SINS, next they are used at calculating the initial conditions for the mode of bringing the $\mathrm{SC}$ orientation to the one specified in the ORF.
At $t \geq t_{1}^{*}$ the measured variables $\Lambda_{k}^{\circ}, \omega_{k}^{o}, \varepsilon_{k}^{o}$ and $\Lambda_{k}, \boldsymbol{\omega}_{k}$ are applied for calculating the values $\boldsymbol{\sigma}_{k}=$ $\mathbf{e}_{k} \operatorname{tg}\left(\Phi_{k} / 4\right), \mathbf{E}_{k}, \mathcal{E}_{k}, \mathbf{C}_{k}^{\mathrm{e}} \equiv \mathbf{C}\left(\mathcal{E}_{k}\right), \boldsymbol{\sigma}_{k}^{\mathrm{e}}=\mathbf{e}_{k}^{\mathrm{e}} \operatorname{tg}\left(\Phi_{k}^{\mathrm{e}} / 4\right)$, $\boldsymbol{\omega}_{k}^{\mathrm{e}} \equiv \delta \boldsymbol{\omega}_{k}=\boldsymbol{\omega}_{k}-\mathbf{C}_{k}^{\mathrm{e}} \boldsymbol{\omega}_{k}^{\mathrm{o}}, \delta \boldsymbol{\phi}_{k}$. That allows to calculate the RW cluster digital control vector by main relation

$$
\mathbf{M}_{k}^{\mathrm{r}}=\boldsymbol{\omega}_{k} \times \mathbf{G}_{k}^{\mathrm{o}}+\mathbf{J}\left(\mathbf{C}_{k}^{\mathrm{e}} \boldsymbol{\varepsilon}_{k}^{\mathrm{o}}+\left[\mathbf{C}_{k}^{\mathrm{e}} \boldsymbol{\omega}_{k}^{\mathrm{o}} \times\right] \boldsymbol{\omega}_{k}+\tilde{\mathbf{m}}_{k}\right)
$$

where vector $\mathbf{G}_{k}^{o}=\mathbf{J} \boldsymbol{\omega}_{k}+\boldsymbol{H}_{k}$ and the vector $\tilde{\mathbf{m}}_{k}$ is formed in accordance with the following two stages:

1) $\forall t \in\left[t_{1}^{*}, t_{2}^{*}\right)$ while $\Phi^{\mathrm{e}}(t)>\Phi_{* 2}^{\mathrm{e}} \equiv \Phi^{\mathrm{e}}\left(t_{2}^{*}\right)$ for a given value $\Phi_{* 2}^{\mathrm{e}}$ at any time $t=t_{2}^{*}$, vector $\tilde{\mathbf{m}}_{k}=\tilde{\mathbf{u}}_{k}^{\mathrm{e}}$ is calculated for error in MPR vector $\sigma^{\mathrm{e}}$ in the form

$$
\tilde{\mathbf{u}}_{k}^{\mathrm{e}}=-\left[\mathbf{D}\left(\boldsymbol{\sigma}_{k}^{\mathrm{e}}\right)\left(k_{\sigma}^{d} \boldsymbol{\sigma}_{k}^{\mathrm{e}}+\mathbf{b}\left(\boldsymbol{\sigma}_{k}^{\mathrm{e}}, \boldsymbol{\omega}_{k}^{\mathrm{e}}\right)\right)+k_{\omega}^{d} \boldsymbol{\omega}_{k}^{\mathrm{e}}\right],
$$

but taking into account general restrictions on modules of vectors $\boldsymbol{\omega}_{k}$ and $\mathbf{u}_{k}$ in algorithm A;

2) at the notation $\boldsymbol{\epsilon}_{k}=-\delta \boldsymbol{\phi}_{k}$, the digital vector $\tilde{\mathbf{m}}_{k}$ is implemented $\forall t \geq t_{2}^{*}$ as follows

$$
\mathbf{g}_{k+1}=\mathbf{B g} \mathbf{g}_{k}+\mathbf{C} \boldsymbol{\epsilon}_{k} ; \tilde{\mathbf{m}}_{k}=\mathbf{K}\left(\mathbf{g}_{k}+\mathbf{P} \boldsymbol{\epsilon}_{k}\right),
$$

where $\mathbf{B}, \mathbf{C}, \mathbf{P}$ and $\mathbf{K}$ are constant matrices.

\section{The Results of Computer Simulation}

Let's assume that a mini-satellite with a mass of 250 $\mathrm{kg}$ is placed in a sun-synchronous orbit with the altitude of $600 \mathrm{~km}$, inclination of $97.787 \mathrm{deg}$ and ascending node longitude of $30 \mathrm{deg}$, and at the time $t_{0}=0$ the SC is flying over the orbit's ascending node when separating from a booster with the angular rate vector module $\omega_{0}=3 \mathrm{deg} / \mathrm{s}$. Assume also that MD has the constraint $\mathrm{l}^{\mathrm{m}}=10 \mathrm{Am}^{2}$ for components of the EMM vector, the period $T_{u}^{\mathrm{m}}=4 \mathrm{~s}$ of its digital control, and in control law of the RW cluster with period $T_{u}=0.25 \mathrm{~s}$, restrictions $\omega^{\mathrm{m}}=1 \mathrm{deg} / \mathrm{s}, \mathrm{u}^{\mathrm{m}}=0.3 \mathrm{deg} / \mathrm{s}^{2}$ in the reference model, the coefficients $k_{\sigma}^{d}$ and $k_{\omega}^{d}$ were calculated for parameters $T_{r}=60 T_{u}$ and $\xi=0.95$.

In Fig. 2 we present the simulation results for the angular velocity vector $\boldsymbol{\omega}(t)$ of mini-satellite at its digital control by both the MD and the RW cluster in all initial orientation modes.

For given value $\omega_{1}^{*}=0.5 \mathrm{deg} / \mathrm{s}$, the time $t_{1}^{*}=6336 \mathrm{~s}$ of the SC's calming mode end is defined automatically, as well as the values $\boldsymbol{\Lambda}_{* 1}, \boldsymbol{\omega}_{1}^{*}, \boldsymbol{\sigma}_{* 1}^{\mathrm{e}}=\mathbf{e}_{* 1}^{\mathrm{e}} \operatorname{tg}\left(\Phi_{* 1}^{\mathrm{e}} / 4\right)$ with $\Phi_{* 1}^{\mathrm{e}}=175.56 \mathrm{deg}$ and $\boldsymbol{\omega}_{* 1}^{\mathrm{e}}=\boldsymbol{\omega}_{1}^{*}-\mathbf{C}_{* 1}^{\mathrm{e}} \boldsymbol{\omega}_{* 1}^{\mathrm{o}}$. Changing the vectors $\mathbf{L}$ and $\mathbf{M}^{\mathrm{m}}$ of the $\mathrm{MD}$ in this mode are presented in Figs. 3 and 4. For given value $\Phi_{* 2}^{\mathrm{e}}=0.083 \mathrm{deg}=300$ arcsec, vector law $\tilde{\mathbf{m}}$ in (5) is switching from (6) to (7) at the time moment $t_{2}^{*}=6583.6 \mathrm{~s}$.

Changing the angles $\phi_{1}$ (roll, blue), $\phi_{2}$ (yaw, green), $\phi_{3}$ (pitch, red) and the angle $\Phi^{\mathrm{e}}$ (black) of the SC rotation in ORF $\forall t \geq t_{1}^{*}=6336 \mathrm{~s}$ are presented in Fig. 5, and some details in changing the vectors $\omega, \mathbf{M}^{\mathrm{r}}$ and $\mathbf{m}$ at bringing the SC orientation to the ORF - in Figs. 6, 7 and 8. At last, in Figs. 9 and 10 we present the errors in angular velocities $\delta \omega_{i}$ and angles $\delta \phi_{i}$ at the ACS transition to the steady-state mode of the SC angular stabilization. 


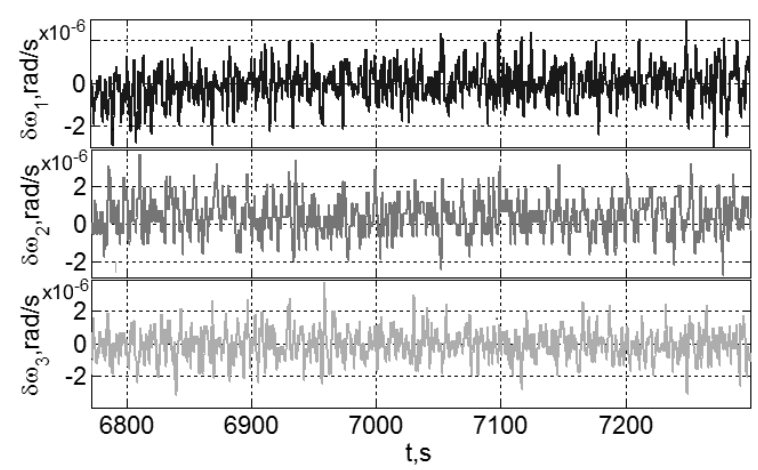

Figure 9. Changing the vector $\delta \boldsymbol{\omega}$ in the stabilization mode

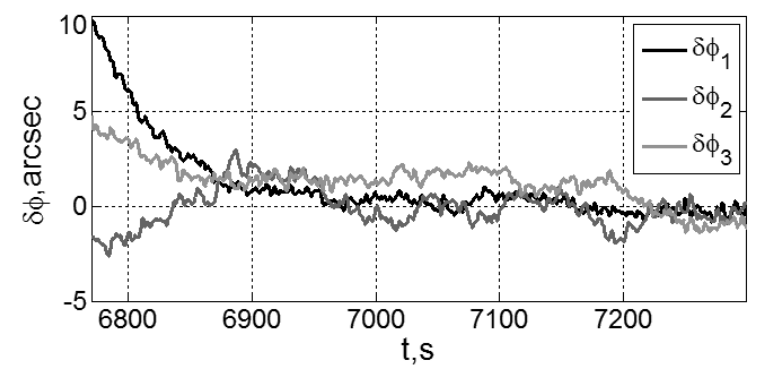

Figure 10. Changing the vector $\delta \phi$ in the stabilization mode

Here, all noises in measurements and disturbing torques were taken into account, but careful filtering of measurements and selection of parameters for autonomous digital control laws allowed to achieve good results on the mini-satellite's ACS accuracy during the mini-satellite initial orientation modes.

\section{Conclusions}

Autonomous angular guidance and modularly limited vector digital control using a vector of the modified Rodrigues parameters are applying to bring the spacecraft's orientation from completely arbitrary to the required one.

Autonomous vector digital control laws of a three-axis magnetic drive and a minimally redundant cluster of the reaction wheels (flywheels) are applying to calm down of a mini-satellite's somersaulting after its separating from a booster and to bring its orientation to a given position in the orbital reference frame without any propulsion unit.

The paper's main new breakthroughs are the following:

(i) autonomous vector digital control of the flywheel cluster with explicit distribution of control torque vector between flywheels taking into account limited resources of the cluster on vectors of control torque and angular momentum;

(ii) unloading the flywheel cluster from accumulated angular momentum using magnetic drive with its digital control by original compensation scheme; (iii) built-in identification and compensation of a dry friction torque on a rotation axis of each reaction wheel.

The developed methods and algorithms for autonomous guidance and digital control of mini-satellite in the initial orientation modes are presented, as well as the results of computer simulation taking into account all noises in measurements and disturbing torques. These results were demonstrated good accuracy of the minisatellite's attitude control system, achieved by careful discrete filtering of measurements and choice of parameters in simple digital control laws.

The developed algorithms for autonomous guidance and digital control of geodetic mini-satellites are simple, reliable, and implementable in space technology [Testoyedov et al., 2017].

\section{Acknowledgements}

The work was supported by Russian Foundation for Basic Research, Grant no. 20-08-00779-a.

\section{References}

Aleksandrov, A. and Tikhonov, A. (2018). Rigid body stabilization under time-varying perturbations with zero mean values. Cybernetics and Physics, 7(1), pp. 5-10.

Materassi, M. and Morrison, P. (2018). Metriplectic torque for rotation of a rigid body. Cybernetics and Physics, 7 (2), pp. 78-86.

Smirnov, Y. (1981). Some problems of mathematical control theory. Leningrad University Press, Leningrad.

Somov, Y. (1996). Feedback linearization and VLF techniques on the synthesis of spacecraft's gyromoment attitude control systems. In IEEE Intern. Conf. on Systems, Man and Cybernetics. Information Intelligence and Systems, vol. 4, Beijing, pp. 2522-2527.

Somov, Y., Butyrin, S., and Somov, S. (2019). Guidance and control of a space robot-manipulator at approach and capturing a passive satellite. IFAC-PapersOnLine, 52 (12), pp. 538-543.

Somov, Y., Butyrin, S., Somova, T., and Somov, S. (2018). In-flight verification of attitude control system for a land-survey satellite at a final of its manufacturing. IFAC-PapersOnLine, 51 (30), pp. 66-71.

Somov, Y., Butyrin, S., Somova, T., and Somov, S. (2021). Health checking autonomous attitude control system of Earth-observing miniature satellite in initial orientation modes. IEEE Journal on Miniaturization for Air and Space Systems, 2 (2), pp. 51-58.

Testoyedov, N., Rayevsky, V., Somov, Y., Titov, G., and Yakimov, Y. (2017). Attitude and orbit control systems of Russian communication, navigation and geodesic satellites: History, present and future. IFACPapersOnLine, 50 (1), pp. 6422-6427. 